\title{
Enlarge Bandwidth of Multimedia Server with Network Attached Storage System
}

\author{
Dan Feng, Yuhui Deng, Ke Zhou, Fang Wang \\ Key Laboratory of Data Storage System, Ministry of Education \\ College of Computer, Huazhong University of Science and Technology \\ Wuhan 430074, China \\ dfeng@hust.edu.cn
}

\begin{abstract}
Network attached storage system is proposed to solve the bottleneck problem of the multimedia server. It adds a network channel to the RAID and data can be transferred between the Net-RAID and clients directly. The architecture avoids expensive store-and-forward data copying between the multimedia server and storage devices when clients download/upload data from/to the server. The system performance of the proposed architecture is evaluated through a prototype implementation with multiple network disk arrays. In multi-user environment, data transfer rate is measured 2 3 times higher than that with a traditional disk array, and service time is about 3 times shorter. Experimental results show that the architecture removes the server bottleneck and dynamically increases system bandwidth with the expansion of storage system capacity.
\end{abstract}

\section{Introduction}

Multimedia service is pervasive on the Internet now and continues to grow rapidly. Most multimedia service provider systems have adopted a typical system architecture in which the storage devices are attached privately to the server. ${ }^{[1],[2]}$ When a client browses some multimedia data from the server, data should be fetched from the storage devices and then forwarded to the client by the server. Unfortunately, with the steady growth of Internet subscribers, the multimedia server quickly becomes a system bottleneck.

More recently, there have been some research efforts invested in solving the bottleneck problem of the multimedia servers. A distributed server architecture that places the streaming servers close to the user clusters has been proposed, ${ }^{[3]}$ where the system is able to achieve scalable storage and streaming capacities by introducing more repository servers and local servers as the traffic increases. A scalable multimedia server based on a clustered architecture is discussed, ${ }^{[4]}$ where a group of nodes are connected by a switch (interconnection network). These related works have made it progressive to enhance the aggregate bandwidth of the multimedia system.

In this paper, Network attached Redundant Arrays of Independent Disks(NetRAID) is proposed to solve the server bottleneck. There are two different channels in the disk array. One is SCSI (Small Computer System Interface) bus to make the disk 
array work as a normal storage system. And the other is network interface to transfer data between clients and the disk array directly. A multimedia server with Net-RAIDs is implemented and the experimental results show that the bandwidth of the server is enlarged by the Net-RAIDs.

\section{Architecture of Multimedia Server System with Net-RAIDs}

A multimedia server is designed with the network attached RAID. It is shown in Fig.1. All Net-RAIDs are centrally controlled by the server through the SCSI channel for the convenience of management just like a normal storage system, while all network interfaces of Net-RAIDs are allowed parallel data transmission. By keeping the SCSI channel of Net-RAID connected to the multimedia file server to exert central control, it strikes a good balance between a centralized file management and a distributed data storage.

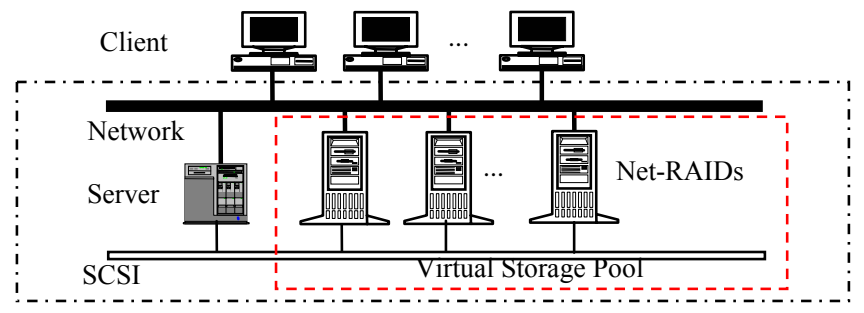

Fig. 1. Multimedia Server with Net-RAIDs

Storage system capacity must keep pace with the continuous growth of multimedia data. The system in Fig. 1 achieves this capacity scalability by expanding the system storage capacity incrementally with additional Net-RAIDs along with associated network interfaces that expand data transmission rate proportionally.

\section{The Redirection of Data Transfer}

All Net-RAIDs in the system are collected as a virtual storage pool. The virtual storage protocol consists of virtual layer, logic map layer and data redirection layer. The virtual layer is used to simulate a standard block device driver and register the virtual storage pool. The logic map layer provides standard interface with block buffer cache and realizes the address map of the virtual storage pool. Different logic map layer leads to different virtual storage pool functions. The data redirection layer provides an interface for physical device drivers, and redirects the data requests from the host to the physical devices.

For example, a File Transfer Protocol(FTP) session consists of two connections. One is control connection for a client to connect with the server and it is kept in the whole session. Another is data connection for the server to transfer data with a client and it is established when data should be downloaded/uploaded to the server. Because 
FTP uses different logical channels to transport control and data packet, we move the logical channel of data connection from the server to the physical network channel of Net-RAID.

When the client downloads a file, the server parses the data information (start address and data length) of the requested file over SCSI channel. Afterwards, the server sends the data information and client information to Net-RAID over the network. A data connection is established between the Net-RAID and the client. Net-RAID gets the requested data from SCSI disks in terms of the data information, and transfers the data to the client directly according to the client information.

\section{Performance Measurement}

In order to get a performance comparison between the prototype and the traditional system where the disk array is only attached to the server, we configure a HUSTRAID $^{[6]}$ that has the same hardware platform as Net-RAID, except for NIC. The HUST-RAID is directly attached to the multimedia server through the SCSI channel. Peak read and write performances of HUST-RAID are $46 \mathrm{MB} / \mathrm{s}$ and $33 \mathrm{MB} / \mathrm{s}$, respectively. The multimedia server is configured as a FTP server and it is connected to the $100 \mathrm{Mbps}$ Ethernet.

The performance of the system is measured by the aggregate bandwidth when a number of clients download/upload files from/to the server simultaneously. Table 1 shows the performance comparison between the prototype and the traditional system. The aggregate bandwidth of the prototype is larger than that of the traditional one and it approaches the network bandwidth. In multi-user environment, the data transfer rate is $2 \sim 3$ times higher than that with a traditional disk array.

Table 1 Performance comparison between the prototype and the traditional system

\begin{tabular}{|c|c|c|c|c|c|c|c|}
\hline \multirow{2}{*}{ operation } & \multirow{2}{*}{$\begin{array}{l}\text { Number } \\
\text { of } \\
\text { clients }\end{array}$} & \multicolumn{3}{|c|}{ Traditional System } & \multicolumn{3}{|c|}{ Prototype system with Net-RAID } \\
\hline & & $\begin{array}{l}\text { Data } \\
\text { transfer } \\
\operatorname{rate}(\mathrm{MB} / \mathrm{s})\end{array}$ & $\begin{array}{l}\text { Average } \\
\text { rate } \\
(\mathrm{MB} / \mathrm{s})\end{array}$ & $\begin{array}{l}\text { Aggregate } \\
\text { bandwidth } \\
(\mathrm{MB} / \mathrm{s})\end{array}$ & $\begin{array}{l}\text { Data } \\
\text { transfer } \\
\text { rate(MB/s) }\end{array}$ & $\begin{array}{l}\text { Average } \\
\text { rate } \\
(\mathrm{MB} / \mathrm{s})\end{array}$ & $\begin{array}{l}\text { Aggregate } \\
\text { bandwidth } \\
(\mathrm{MB} / \mathrm{s})\end{array}$ \\
\hline \multirow{10}{*}{$\begin{array}{l}\text { Download } \\
\text { File }\end{array}$} & 1 & 6.75 & 6.75 & 6.75 & 7.36 & 7.36 & 7.36 \\
\hline & \multirow{2}{*}{2} & 2.83 & \multirow{2}{*}{2.62} & \multirow{2}{*}{5} & 4.82 & \multirow{2}{*}{4.67} & \multirow{2}{*}{9.33} \\
\hline & & 2.41 & & & 4.51 & & \\
\hline & \multirow{3}{*}{3} & 1.28 & \multirow{3}{*}{1.25} & \multirow{3}{*}{3.76} & 3.28 & \multirow{3}{*}{3.34} & \multirow{3}{*}{10.01} \\
\hline & & 1.12 & & & 4.01 & & \\
\hline & & 1.36 & & & 2.72 & & \\
\hline & \multirow{4}{*}{4} & 0.88 & \multirow{4}{*}{0.82} & \multirow{4}{*}{3.29} & 2.62 & \multirow{4}{*}{2.48} & \multirow{4}{*}{9.93} \\
\hline & & 0.93 & & & 2.35 & & \\
\hline & & 0.76 & & & 3.11 & & \\
\hline & & 0.72 & & & 1.85 & & \\
\hline
\end{tabular}

When we add another Net-RAID to the prototype system, the aggregate bandwidth is nearly $20 \mathrm{MB} / \mathrm{s}$ (see Table 2 ). It shows that the performance of the system increases almost linearly with the increase of the number of Net-RAIDs, and the system bottleneck has been removed from the server to network. 
Table 2 Performance comparison when two Net-RAIDs are in the system

\begin{tabular}{|c|c|c|c|c|c|}
\hline \multirow[b]{2}{*}{ Operation } & \multirow{2}{*}{$\begin{array}{l}\text { Number } \\
\text { of clients }\end{array}$} & \multicolumn{2}{|c|}{ Traditional Architecture } & \multicolumn{2}{|c|}{$\begin{array}{c}\text { Prototype system with Net- } \\
\text { RAID }\end{array}$} \\
\hline & & $\begin{array}{l}\text { Data transfer } \\
\operatorname{rate}(\mathrm{MB} / \mathrm{s})\end{array}$ & $\begin{array}{l}\text { Aggregate } \\
\text { bandwidth } \\
\text { (MB/s) }\end{array}$ & $\begin{array}{l}\text { Data transfer } \\
\text { rate( }(\mathrm{MB} / \mathrm{s})\end{array}$ & $\begin{array}{l}\text { Aggregate } \\
\text { bandwidth } \\
(\mathrm{MB} / \mathrm{s})\end{array}$ \\
\hline \multirow{3}{*}{$\begin{array}{l}\text { Download } \\
\text { File }\end{array}$} & \multirow{3}{*}{3} & 3.23 & \multirow{3}{*}{9.94} & 6.67 & \multirow{3}{*}{19.03} \\
\hline & & 3.32 & & 6.85 & \\
\hline & & 3.57 & & 5.51 & \\
\hline \multirow{3}{*}{$\begin{array}{l}\text { Upload } \\
\text { File }\end{array}$} & \multirow{3}{*}{3} & 4.50 & \multirow{3}{*}{10.10} & 6.37 & \multirow{3}{*}{18.40} \\
\hline & & 2.96 & & 5.35 & \\
\hline & & 2.64 & & 6.68 & \\
\hline
\end{tabular}

\section{Conclusions}

An innovative network attached Disk array architecture, called Net-RAID, is proposed and implemented. It adds a network channel to the RAID and data can be transferred between the Net-RAID and clients directly. The architecture removes the server bottleneck and dynamically increases system bandwidth with the expansion of storage system capacity. Experimental results provide useful insights into the performance behavior of the system. The architecture can also be adopted to transfer massive data in other different servers, such as database server, HTTP server and so on.

\section{Acknowledgements}

This research is supported by National Nature Science Foundation of China (No. 60273074), and the Special Foundation of Excellent Ph.D. Thesis of China.

\section{References}

1. H. Radha, Y. Chen, K. Parthasarathy, R. Cohen: Scalable Internet Video Using MPEG-4, Image Communications, 15, 1999.

2. J. Pieper, S .Srinivasan, B. Dom: Streaming-Media Knowledge Discovery, IEEE Computer, Vol. 34, No. 9, (2001) 68-74

3. SA Barnett and GJ Anido: A cost comparison of distributed and centralized approaches to video-on-demand, IEEE J. Select. Areas Commun., vol. 14. (1996) 1173-1183

4. R. Tewari, D. Dias, R. Mukherjee, H. Vin: High Availability for Clustered Multimedia Servers, Proc. Int. Conf. Multimedia Computing and Systems, Tokyo, (1996) 144

5. Milind Buddhikot, Gurudatta, Parulkar, and Jerome, Cox, Jr.: Design of a Large Scale Multimedia Storage Server. Journal of Computer Networks and ISDN Systems. Elsevier, North Holland (1994) 504-524

6. Cheng peng, Jiangling Zhang, Hai Jin "Design of High Performance RAID in Real-Time system". ACM, Computer Architecture News, Vol. 27. (1999) 